\title{
viewpoint
}

\section{A tale of two strategies}

\author{
The moral imperative to tackle ageing
}

\section{Colin Farrelly}

\author{
"Do not go gentle into that good night, \\ Old age should burn and rave at close of day; \\ Rage, rage against the dying of the light." \\ Dylan Thomas, 1951
}

$\mathrm{R}$ esearch into ageing is a fascinating field of scientific study, not least because it addresses a topic that, sooner or later, affects every one of us. At the same time, the science itself is rapidly progressing with a constant flow of publications that help to elucidate the numerous causes of ageing, such as DNA damage, the shortening of telomeres and oxidation processes in the cell. On the basis of this wealth of information, scientists have started to explore interventions that could modify the biological processes that lead to ageing, thereby creating opportunities for people to live longer and healthier lives. However, the biology of ageing is complex and involves many molecular and physiological processes that, although they eventually lead to ageing, still have important functional roles. It is therefore not surprising that, although scientists basically agree that human ageing itself is not immutable, they continue to disagree as to what might constitute the most promising strategy for retarding it. Such scientific disagreement is, of course, neither new nor specific to ageing-related research - it rightly permeates all branches of scientific inquiry.

However, another disagreement now embroils researchers studying ageing: the debate about how they should frame the moral imperative to retard human ageing. Is ageing actually a disease? If so, should we invest more public money to find a cure for it, or are the medical interventions that could retard ageing best classified as 'enhancements' rather than therapies? Does this really matter and, in any case, do the answers to these questions have an impact on the prioritization of research into human ageing?

This ethical and social debate is mostly confined to the field of biogerontology. Scientists who are working on applied research to find treatments for cancer or Alzheimer disease, for example, seldom find themselves pressed to offer a rationale for why they are doing so: it is apparently self-evident that a cure for cancer or Alzheimer disease is desirable and important. Yet, research into ageing pushes the medical orthodoxy in ways that research into treating a particular disease does not. This explains the interesting and spirited debates among biogerontologists about how to pitch the importance of their research to the general public and policy makers, and how to make a compelling case for diverting a greater share of public funding into ageing-related research.

Research into ageing certainly appears to be woefully underfunded: in 2006, the overall budget of the US National Institutes of Health (Bethesda, MD, USA) was US\$28 billion, but less than $0.1 \%$ was spent on understanding the biology of ageing, and how it predisposes us to an array of debilitating diseases and disorders in the later stages of our lives (Olshansky et al, 2006).

On one side of this debate are those who claim that ageing is itself a disease and should be treated as such. Arthur Caplan, a bioethicist at the University of Pennsylvania (Philadelphia, PA, USA), is one such proponent. He argues that: "Disease is almost always defined as any pathological change in the body. Pathological change is inevitably defined as constituting any morbid process in the body. And morbid processes are usually defined in terms of disease states of the body. Regardless of the circularity of this concept, ageing would therefore seem to have a prima facie claim to being counted as a disease" (Caplan, 2005). On the other side of the debate are biodemographers, such as Jay Olshansky at the University of Illinois (Chicago, IL, USA), who emphasize the potential health and socio-economic benefits of retarding ageing-if by only a few years - even if it is not considered a de facto disease.

$\mathrm{T}$ he first argument is a powerful one: if we classify ageing as a human disease, it is undeniably the most prevalent with a statistical frequency of $100 \%$ (Caplan, 2005). Aubrey De Grey, who is a vocal advocate of defeating ageing, argues that 100,000 people worldwide die each day from age-related causes - in industrialized countries, approximately $90 \%$ of all deaths are caused by ageing (De Grey \& Rae, 2007). Therefore, if we accept that ageing is a disease, the obvious prescription is to find a treatment- - thereby making ageingrelated research a priority—and De Grey argues that those who hesitate to launch a 'war on ageing' are further delaying a cure (De Grey, 2005a).

\section{...research into ageing pushes the medical orthodoxy in ways that research into treating a particular disease does not}

The arguments put forward by De Grey have led to a point of genuine contention between him and his critics: what constitutes a proportionate response to ageing? Once ageing is given disease status, it will dwarf all competing claims for biomedical research funding because, more than any other factor-at least in the industrialized 
world-ageing has an adverse impact on the health of everyone. Ageing increases the risk of cancer, diabetes, Alzheimer disease, dementia, infection, infertility, bone fracture and, ultimately, death. Critics of De Grey believe that it is irresponsible to promise or aspire to a cure for ageing, and have described his Strategies for Engineered Negligible Senescence (SENS) programme as "a confused mixture, or hodgepodge" (Warner et al, 2005).

Putting aside the disagreements over the specifics of the proposed strategy, the claim made by De Grey that there is a stringent moral imperative to tackle ageing remains valid. If it is correct to give ageing the label of a disease, then we do not need all the specifics of a potential cure at hand before we can set out to do something about it. No one lambasted US President Richard Nixon for declaring "war on cancer" in the 1970s without having an established scientific consensus about how cancer could be cured. What really mattered was the fact that cancer killed, and still kills, millions of people worldwide-by investing more money and resources into cancer research, we hope to reduce the annual death and morbidity toll, and the crippling socio-economic costs that go along with the disease, no matter whether a scientific consensus exists or not.

Are the calls for a war on ageing premature or inappropriate, and how much science do we need before we can justifiably launch such a war? The appropriate threshold should depend on the magnitude of the likely benefits that a cure could yield and the costs of inaction. Given the high stakes, and because we know with certainty what the costs of inaction will be, one could make a strong case that we should not let our uncertainty about the likelihood of success impede our efforts. This argument is often used effectively to galvanize action to combat climate change, and investing in research for a cure for ageing has an even more sound scientific basis: we are more certain that ageing is responsible for death and disease worldwide than we are that climate change might be. Furthermore, setting the scientific bar too high is self-defeating-we need investment in basic research into ageing before we can speculate about applications and cures. If researchers could find an easy or cheap cure then they would have done so already. Therefore, without adequate investment, we will never know whether we can realistically reap the health dividends that ageing-related research could yield.
$\mathrm{H}$ owever, if the consensus is that the idea of defeating ageing (De Grey, 2005b) is utopian and unrealistic_as many scientists currently believe-this will have an impact on how important we think tackling ageing is. The magnitude of the likely benefits that are promised to come from research aimed at defeating ageing is greater than that expected to come from merely retarding it. In effect, promising to deliver an extra 1,000 years of healthy life matters little if the likelihood of being able to deliver the benefits is nil-'hyping' the benefits is self-defeating. The disagreement over the science therefore explains much of the disagreement between those who champion the hard stance of De Grey on fighting ageing and those who believe that his position does more harm than good in promising too much. Furthermore, in a world in which poverty and infectious disease afflict so many, making research into ageing the top priority, rather than research into malaria, for example, is unfair to those for whom the chance to suffer from old age would be a luxury. This is another reason why some believe that the 'ageing is a disease, we should find a cure' strategy is inherently and ethically flawed.

Classifying ageing as a disease also has inherent social problems because it might worsen existing negative attitudes towards the elderly. We must consider, for example, how to deal with the interests of those who are already in the late stages of old age: should they be treated differently now that we know they are 'ill'? This is similar to the concerns of the advocates of rights for the disabledlabelling ageing as a disease might be seen as an affront to the equality of the aged. One might object to the idea of 'ageing is a disease' by invoking what Buchanan and co-authors (2000) call the expressivist objection, which maintains that aspirations to eliminate senescence entail attitudes that compromise the equal respect and concern of the aged. These negative judgements could divert attention away from aiding those who are currently alive and aged towards speculative preventative measures that seek to retard or eliminate ageing.

However, the expressivist objection is flawed because it confuses negative judgements about the attributes of ageing itself with judgements about aged individuals:

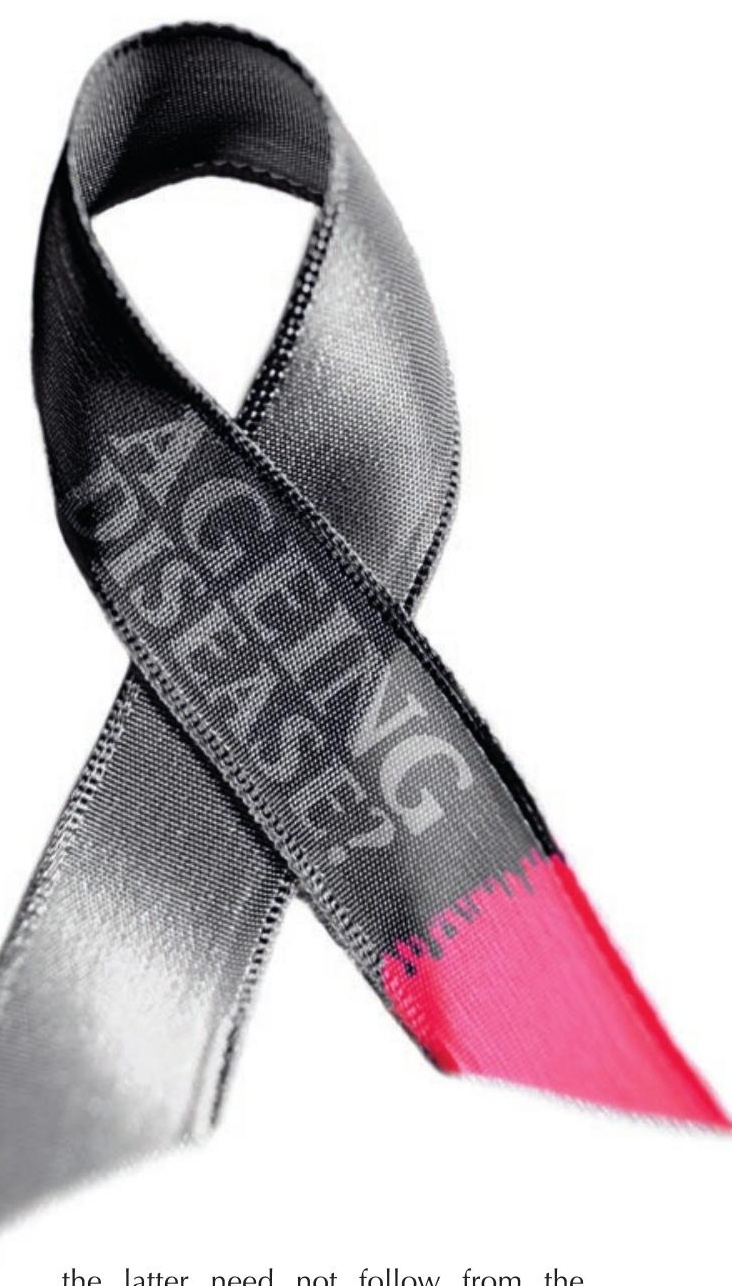

the latter need not follow from the former. The aspiration to retard ageing, even when it is motivated by the belief that ageing is a disease, does not mean that one must view those who are aged as less than human. The same applies to the aspiration to prevent all other diseases and disadvantages. Parents who encourage their children to wear bicycle helmets do so because they want to minimize the risk of head injuries. The fact that parents seek to avoid mental impairment caused by bicycle accidents does not mean that they disrespect those who are currently living with mental impairments. The same applies to ageing. Parents who hope their children will not be afflicted with the same diseases and disabilities that they see their own parents suffering from are not making negative judgements about the elderly and infirm themselves. If those who favour waging a war against ageing also make the claim that the interests of those who are now disadvantaged by ageing have no moral weight, then their position is indeed guilty of violating the equality and respect of the aged. However, that is not a premise of the 'ageing is a disease, we should find a cure' argument. 


\section{Once ageing is given disease status, it will dwarf all competing claims for biomedical research funding because [...] ageing has an adverse impact on the health of everyone}

Nonetheless, there is a risk that if, as notedabove, the potential benefits of ageingrelated research are over-inflated to a great extent, then all other laudable social aims might be reduced to the status of mere trivialities. This is why it is imperative to pursue a proportionate response to ageing. The real challenge facing those who embrace the 'waging war on ageing' slogan is that they must take seriously the legitimate concerns of inclusion and proportionality. Such concerns require a nuanced public ethic that is not easily captured in catchy slogans.

\section{$\mathrm{T}$} he second-and purportedly more modest-strategy for developing a moral argument in favour of tackling ageing seeks to reveal the folly of deliberately limiting our understanding of what constitutes medical treatment. We currently adhere to the impoverished conceptual framework of the therapy/enhancement distinction, which is the foundation of the influential account of just health care by Norman Daniels, for example (Daniels, 1985). According to Daniels, professor of ethics and population health at the Harvard School of Public Health (Cambridge, MA, USA), "the primary rationale for claiming that we are obligated to provide people with medical service is that it meets an important need for treatment of disease or disability" (Daniels, 2000). Yet even Daniels acknowledges that the treatment/enhancement distinction does not map unqualifiedly onto the moral boundary between obligatory and non-obligatory services (Daniels, 2000).

Harry Moody, Director of Academic Affairs for the American Association of Retired Persons (AARP; Washington, DC, USA), argues that interventions to retard ageing could make substantial progress in reducing mortality and morbidity in the same way that medical interventions have done in childbirth: "we've made a lot of progress over the last century or more in redefining childbirth as an area where we could reduce mortality, reduce morbidity, achieve a lot of good things, and we need to do that for the process of ageing" (Moody \& Caplan, 2004). Immunizations are another example of how non-therapeutic medical interventions have come to be considered as medical necessities (Olshansky et al, 2006).

To make a pressing case for investing a greater portion of scarce public funds into ageing-related research, rather than into research for particular diseases, the advocates of this second strategy emphasize the magnitude of the health dividends that would be conferred by retarding human ageing. For example, Olshansky and coauthors claim in their paper 'In Pursuit of the Longevity Dividend" that a modest retardation of human ageing by just seven years could confer benefits greater than those that could be achieved by the elimination of cancer (Olshansky et al, 2006). This strategy does not invoke the idea that ageing is a disease, but rather focuses exclusively on the likely health and socio-economic benefits that could be reaped by modifying the biological processes of ageing.

At first glance, this second strategy has the appeal of avoiding the stigma that might come with labelling ageing as a disease. Yet, as noted above, the latter strategy can negate this objection by distinguishing between a judgement about ageing itself and a judgement about those persons who are afflicted with ageing. The question then is whether the second strategy confers any distinctive benefits that the 'ageing is a disease' position cannot. One genuine benefit is that it allows us to take a much more provisional stance on the stringency of the imperative to tackle ageing. Proponents of the 'longevity dividend' do not call for abandoning the current approach of tackling one disease at a time - rather, they advocate supplementing this with a broader strategy that seeks to retard ageing. Unlike the call for waging a war on ageing itself, the advocates of this second approach call for greater caution when it comes to the promises made about what research into ageing will deliver in the foreseeable future.

$\mathrm{H}$ owever, the aspiration to find a reasonable balance between tackling ageing itself and the diseases that are associated with ageing stands a chance of being proportionate only if all of the advocates of scientific research are playing by the same rules - in terms of the promises made and timescales estimated when appealing for public funding. This is where the second strategy appears to be too conservative and, hence, problematic. To achieve proportionality, policy-makers and the general public need a sense of the magnitude and likely timescales of the benefits that could be delivered by intervening with the biological processes of ageing versus those promised by the strategy of tackling one disease at a time. The greater the magnitude of these benefits-even if one abandons the 'ageing is a disease' premise- - the greater the portion of public funds that should be invested in ageing-related research.

\section{...inflating the potential benefits of ageing research will collapse the moral landscape to the single collective aspiration to maximize the human lifespan}

To emphasize the modest goal of retarding ageing by only a few years could undervalue the potential benefits of ageing research, as comparable benefits could also be realized by pursuing environmental interventions - such as encouraging a healthier diet and a more active lifestyle. Selling the idea of new and speculative biological interventions in the ageing process will therefore be much more difficult if scientists emphasize a conservative estimate of the likely benefits of ageing research - the appeals for funds to research cancer or Alzheimer disease are not made on equally modest grounds. Proportionality can only be achieved if one considers the possible health benefits of two decades of cancer research versus two decades of research into ageing. The conservative estimates of many researchers who investigate ageing therefore threaten to take things in the opposite direction to the 'ageing is a disease' strategy and could greatly undervalue the importance of ageing-related research. Proportionality requires us to find the middle ground between promising too much and promising too little. Given the meagre amount of public funding that biogerontologists currently receive, it is obvious that the real threat comes from the latter.

S o where does all this leave us? Does a great deal really hinge on the contentious claim that ageing is a disease? It seems that the debate among biogerontologists is primarily a pragmatic one, although it also reflects some disagreement over fundamental moral principles. Those in favour of the 'ageing is a disease' approach believe that it is a necessity, given the dominance of individual diseases in the current funding model. To describe anti-ageing 
interventions as enhancements is to trivialize and jeopardize the enormous benefits that could be reaped by recognizing that ageing itself is the most prevalent of human diseases. Therefore, a modest estimate of the enhancements that research into ageing could confer will not generate the attention necessary for researchers to compete for funding in a system that is dominated by tackling individual diseases.

Yet a downside of this approach is that quantifying the possible benefits is extremely difficult and potentially risky. Talk of defeating ageing therefore threatens to compromise proportionality - in addition to scientific credibility-because it overestimates the likely benefits of research into ageing. Claims of extending the human lifespan by 1,000 years, for example, threaten proportionality because they risk marginalizing all other laudable social goals. If the demands of morality are viewed solely through the lens of cost-benefit analysis, then inflating the potential benefits of ageing research will collapse the moral landscape to the single collective aspiration to maximize the human lifespan.

\section{To describe anti-ageing interventions as enhancements is to trivialize and jeopardize the enormous benefits that could be reaped by recognizing that ageing itself is the most prevalent of human diseases}

However, fairness entails much more than a utilitarian approach prescribes. Prioritarians, for example, believe that the worse-off someone is, the greater the imperative to help him or her (Parfit, 2000). These nuances in our moral sensibilities are jeopardized if one greatly overestimates the benefits of tackling ageing to such an extent that other laudable causes-such as ending poverty or treating early-onset diseases - are viewed as mere distractions from the primary moral objective of maximizing the human lifespan. In such a scenario, the expressivist objection to tackling ageing would have some merit because the aspiration to aid those aged individuals who are now in the last decade of their lives will be seen as trivial compared to the goal of extending, by almost a millennium, the health prospects of future generations.

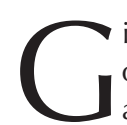
iven that both strategies have their own virtues and vices, it is probably beneficial for biogerontologists to pursue and explore both rather than to invest everything in one or the other. The real importance of tackling ageing should not be contingent on its classification as either a disease or a by-product of our evolutionary history, but rather on its likely impact on the health of individuals and populations. Yet, the task of bringing greater awareness to the potential benefits that research into ageing could confer is a difficult one given the scarcity of resources and the pervasiveness of human disease. Labelling ageing as a disease would allow us to assess whether tackling it gives us more 'bang for our buck' — from a publichealth perspective-than investing in any one of the numerous age-related diseases. Yet, this same message could also be conveyed in the language of 'enhancements' by drawing an analogy with immunizations. Whichever way one frames the issue, it is hard to deny that tackling ageing is a moral and medical emergency-even if the benefits are only 30 or 40 extra years of healthy life, rather than 1,000 years.

However, asserting that the aspiration to retard human ageing is a moral emergency does not mean that the interests of those who are currently afflicted with the pathologies that accompany ageing have no moral weight. Yet, modern medicine is severely limited in terms of the substantive health benefits that it can confer on those who already suffer from these afflictions. Now that we have good scientific evidence for believing that we could alter this situationso that future generations do not suffer the same fate-we have a moral obligation to humanity to respond in a fair and proportionate manner, and to mitigate the biological vulnerabilities that we have inherited from our evolutionary history.

\section{REFERENCES}

Buchanan A, Brock D, Daniels N, Wikler D (2000)

From Chance to Choice: Genetics and Justice.

Cambridge, UK: Cambridge University Press

Caplan A (2005) Death as an unnatural process. EMBO Rep 6: S72-S75

Daniels N (1985) Just Health Care. Cambridge, UK: Cambridge University Press

Daniels N (2000) Normal functioning and the treatment-enhancement distinction. Camb $Q$ Healthc Ethics 9: 309-322

De Grey A (2005a) Life extension, human rights, and the rational refinement of repugnance. J Med Ethics 31: 659-663

De Grey A (2005b) Resistance to debate on how to postpone ageing is delaying progress and costing lives. EMBO Rep 6: S49-S53

De Grey A, Rae M (2007) Ending Aging: The Rejuvenation Breakthroughs That Could Reverse Human Aging in Our Lifetime. New York, NY, USA: St. Martin's

Moody H, Caplan A (2004) Is aging a disease? Sage Crossroads, 22 January. www.sagecrossroads.net Olshansky SJ, Perry D, Miller R, Butler R (2006) In pursuit of the longevity dividend. The Scientist 20: $28-36$

Parfit D (2000) Equality or priority? In The Ideal of Equality, M Clayton, A Williams (eds), pp 81-125. Basingstoke, UK: Palgrave, Macmillan

Warner H et al (2005) Science fact and the SENS Agenda. EMBO Rep 6: 1006-1008

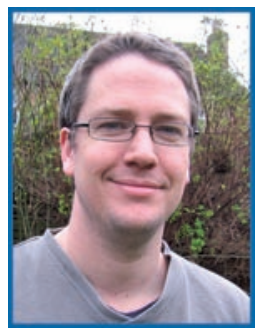

Colin Farrelly is Associate Professor in the Department of Political Science at the University of Waterloo, Ontario, Canada.

E-mail: farrelly@watarts.uwaterloo.ca

doi:10.1038/embor.2008.107 